\title{
RESONANCE OF MINIMIZERS FOR N-LEVEL QUANTUM SYSTEMS WITH AN ARBITRARY COST
}

\author{
Ugo Boscain $^{1,2, *}$ And GrÉGoire Charlot ${ }^{1, \dagger}$
}

\begin{abstract}
We consider an optimal control problem describing a laser-induced population transfer on a $n$-level quantum system. For a convex cost depending only on the moduli of controls (i.e. the lasers intensities), we prove that there always exists a minimizer in resonance. This permits to justify some strategies used in experimental physics. It is also quite important because it permits to reduce remarkably the complexity of the problem (and extend some of our previous results for $n=2$ and $n=3$ ): instead of looking for minimizers on the sphere $S^{2 n-1} \subset \mathbb{C}^{n}$ one is reduced to look just for minimizers on the sphere $S^{n-1} \subset \mathbb{R}^{n}$. Moreover, for the reduced problem, we investigate on the question of existence of strict abnormal minimizer.
\end{abstract}

Mathematics Subject Classification. 49J15, 81V80, 53C17, 49N50.

Received June 25, 2003.

\section{INTRODUCTION}

The problem of designing an efficient transfer of population between different atomic or molecular levels is crucial in many atomic-physics projects $[6,17,20,26,35]$. Often excitation or ionization is accomplished by using a sequence of laser pulses to drive transitions from each state to the next state. The transfer should be as efficient as possible in order to minimize the effects of relaxation or decoherence that are always present. In the recent past years, people started to approach the design of laser pulses by using Geometric Control Techniques (see for instance $[3,16,24,25,32,33])$. Finite dimensional closed quantum systems are in fact left invariant control systems on $S U(n)$, or on the corresponding Hilbert sphere $S^{2 n-1} \subset \mathbb{C}^{n}$, where $n$ is the number of atomic or molecular levels. For these kinds of systems very powerful techniques were developed both for what concerns controllability $[4,18,22,23,34]$ and optimal control $[1,21]$.

The most important and powerful tool for the study of optimal trajectories is the well known Pontryagin Maximum Principle (in the following PMP, see for instance [1,21,31]). It is a first order necessary condition for optimality and generalizes the Weierstraß conditions of Calculus of Variations to problems with non-holonomic

Keywords and phrases. Control of quantum systems, optimal control, sub-Riemannian geometry, resonance, pontryagin maximum principle, abnormal extremals, rotating wave approximation.

1 SISSA-ISAS, via Beirut 2-4, 34014 Trieste, Italy; e-mail: boscain@sissa.it; charlot@sissa.it

2 Département de Mathématiques, Analyse Appliquée et Optimisation, Université de Bourgogne, 9 avenue Alain Savary, BP 47870-21078 Dijon Cedex, France.

* This research has been supported by a Marie Curie Fellowship. Program "Improving Human Research Potential and the Socio-economic Knowledge Base", Contract Number: HPMF-CT-2001-01479.

$\dagger$ Supported by european networks NCN (ERBFMRXCT97-0137) and NACO2 (N.HPRN-CT-1999-00046). 
constraints. For each optimal trajectory, the PMP provides a lift to the cotangent bundle that is a solution to a suitable pseudo-Hamiltonian system.

Anyway, giving a complete solution to an optimization problem (that for us means to give an optimal synthesis, see for instance $[7,9,14,15,30])$ remains extremely difficult for several reasons. First one is faced with the problem of integrating a Hamiltonian system (that generically is not integrable excepted for very special costs). Second one should manage with "non Hamiltonian solutions" of the PMP, the so called abnormal extremals. Finally, even if one is able to find all the solutions of the PMP it remains the problem of selecting among them the optimal trajectories. For these reasons, usually, one can hope to find a complete solution of an optimal control problem in low dimension only.

This paper is the continuation of a series of papers on optimal control of finite dimensional quantum systems $[10,11]$. The main purpose is to show that for a certain class of quantum systems (that contains systems useful for applications) one can reduce remarkably the complexity of the problem. More precisely we prove that for a convex cost depending only on the moduli of controls (e.g. amplitude of the lasers):

- there always exists a minimizer in resonance that connects a source and a target defined by conditions on the moduli of the components of the wave function (e.g. two eigenstates, see Th. 1). Roughly speaking to be in resonance means to use lasers oscillating with a frequency equal to the difference of energy between the levels that the laser is coupling. As a consequence one gets a reduction of the dimension of the problem from $2 n-1$ to $n-1$ ( $n$ being the number of energy levels), see Corollary 1. From a physical point of view this means that one is reduced to look just for the amplitudes of the lasers;

- for the reduced system in dimension $n-1$, we prove that close to any time $t$ of the domain of a given minimizer, there exists an interval of time where the minimizer is not strictly abnormal (see Th. 3). This result is a first step in trying to prove the conjecture that for the reduced problem there are no strictly abnormal minimizer.

This extends some of our previous results (see $[10,11])$.

Here we are considering a class of systems on which it is possible to eliminate the so called drift term. This includes $n$-level quantum systems in the rotating wave function approximation (RWA) and in which each laser couples only close levels. For this kind of systems our reduction is crucial to give a complete solution for systems with $n=3$ for several costs interesting for applications. Moreover it gives some hope to find the time optimal synthesis for systems with four levels and bounded lasers. Finally it is of great help in finding numerical solutions to problems with $n \geq 4$.

The paper is organized as follows. In Section 1.1 we introduce the physical model. In order to characterize controllability, in Section 1.2 we associate a topological graph to the system in a unique way. In Section 1.3 we formulate the optimal control problem, while in Section 1.4 we discuss the costs that are interesting for applications. In Section 1.5 we introduce some key definitions and we state our main questions.

Section 2 is devoted to recall how to eliminate the drift term from the control system, by means of a unitary time-dependent change of coordinates plus a unitary change of controls (interaction picture). This permits to write the system in "distributional" form.

Our main results are stated in Section 3 (see Ths. 1,3). Beside stating that there are always minimizers in resonance and studying strictly abnormal minimizers for the reduced problem, we investigate also the question if every minimizer is in resonance (see Th. 2). More precisely, we state that, under the assumption that the cost function is strictly increasing with respect to the moduli of the controls, every minimizer is weakly-resonant in a suitable sense.

In Section 4 we prove the main results about resonance. To investigate resonance, the key point is to identify the components of the controls responsible of the evolution of the moduli of the components of the wave function. The difficulty comes from the fact that, when a coordinate is zero, the dimension of the "distribution" may fall. This obliges to divide the domain of a minimizer in suitable sub-domains (see Sect. 1.5.1). In the first part of Section 4 we prove our main results, while in Section 4.1 we give an alternative geometrical interpretation. In Section 4.2 we investigate the question if it is possible to join every couple of points in $S^{2 n-1} \subset \mathbb{C}^{n}$ by a 
resonant minimizer. In Section 4.3 we give an example of a minimizer (for a non strictly increasing cost) which is not weakly-resonant and we propose an open question.

The most technical part is Section 5 where we investigate about strictly abnormal minimizers for the reduced problem on the sphere $S^{n-1} \subset \mathbb{R}^{n}$. For trajectories whose coordinates are never zero, our results are just a consequence of the fact that the dimension of the distribution is the same as the dimension of the state space. But again the difficulty is coming from the fall of the dimension when some coordinates are zero.

\subsection{The model}

In this paper we consider closed finite dimensional quantum systems in the rotating wave function approximation (RWA). More precisely we consider systems whose dynamics is governed by the time dependent Schrödinger equation (in a system of units such that $\hbar=1$ ):

$$
\left\{\begin{array}{l}
i \frac{\mathrm{d} \psi(t)}{\mathrm{d} t}=\mathcal{H}(t) \psi(t):=(D+V(t)) \psi \\
\psi(.):=\left(\psi_{1}(.), \ldots, \psi_{n}(.)\right): \mathbb{R} \rightarrow \mathbb{C}^{n}, \quad \sum_{i}\left|\psi_{i}\right|^{2}=1,
\end{array}\right.
$$

where it holds:

(H1) $D=\operatorname{diag}\left(E_{1}, \ldots, E_{n}\right)$ and $V(t)$ is an Hermitian matrix $\left(V(t)_{j, k}=V(t)_{k, j}^{*}\right)$, measurable as function of $t$, whose elements $V(t)_{j, k}$ are either identically zero or controls.

Here $\left({ }^{*}\right)$ indicates the complex conjugation involution. $E_{1}, \ldots, E_{n}$ are the energy levels of the quantum system and the controls $V_{j, k}($.$) , that we assume to be \mathbb{C}$-valued measurable functions, are different from zero only in a fixed interval $[0, T]$. They are connected to the physical parameters by $V_{j, k}(t)=\mu_{j, k} \mathcal{F}_{j, k}(t) / 2, j, k=1, \ldots, n$, with $\mathcal{F}_{j, k}$ the external pulsed fields (the lasers) and $\mu_{j, k}=\mu_{k, j}>0$ the couplings (intrinsic to the quantum system). In the following we say that two levels $E_{j}, E_{k}$ are coupled if $V_{j, k}$ is a control (and not zero). Moreover if all the couplings $\mu_{j, k}$ are equal to a constant $\mu$ (that we normalize to 1 ) we say that the system is isotropic. Otherwise we say that the system is non-isotropic.

Remark 1. The term $D=\operatorname{diag}\left(E_{1}, \ldots, E_{n}\right)$ in equation (1) is called drift and it will be eliminated in Section 2, thanks to the fact that we are in the rotating wave approximation and that each control couples only two levels.

Remark 2. This finite-dimensional problem can be thought as the reduction of an infinite-dimensional problem in the following way. We start with a Hamiltonian which is the sum of a "drift-term" $D$, plus a time dependent potential $V(t)$ (the control term, i.e. the lasers). The drift term is assumed to be diagonal, with eigenvalues (energy levels) $\ldots>E_{3}>E_{2}>E_{1}$. Then in this spectral resolution of $D$, we assume the control term $V(t)$ to couple only a finite number of energy levels by pairs. Let $\left\{E_{j}\right\}_{j \in I}$ (I finite) be the set of coupled levels. The projected problem in the eigenspaces corresponding to $\left\{E_{j}\right\}_{j \in I}$ is completely decoupled and it is described by (1), (H1).

The function $\psi:[0, T] \rightarrow S^{2 n-1} \subset \mathbb{C}^{n}$ solution of the Schrödinger equation (1) is called the wave function. The physical meaning of its components $\psi_{j}(t)$ is the following. For time $t<0$ and $t>T$ (where the controls are zero), $\left|\psi_{j}(t)\right|^{2}$ is the probability of measuring energy $E_{j}$. Notice that, in the intervals of time where $V$ is identically zero, we have:

$$
\frac{\mathrm{d}}{\mathrm{d} t}\left|\psi_{j}(t)\right|^{2}=0, \quad j=1, \ldots n
$$

In the following a state for which we have $\left|\psi_{j}(t)\right|=1$ for some $j$ will be called an eigenstate.

Remark 3. This model is physically reasonable in the case in which:

- the number of energy levels is not too big, and they are distinct by pairs;

- there are not too many couplings between the energy levels. 
The most interesting case is perhaps the one in which only close levels are coupled. In this case $V$ has non null coefficients $V_{j, k}$ only on the second diagonals (for $j$ and $k$ such that $|j-k|=1$ ) and the Hamiltonian reads:

$$
\mathcal{H}(t)=\left(\begin{array}{ccccc}
E_{1} & V_{1,2}(t) & 0 & \cdots & 0 \\
V_{1,2}^{*}(t) & E_{2} & V_{2,3}(t) & \ddots & \vdots \\
0 & V_{2,3}^{*}(t) & \ddots & \ddots & 0 \\
\vdots & \ddots & \ddots & E_{n-1} & V_{n-1, n}(t) \\
0 & \cdots & 0 & V_{n-1, n}^{*}(t) & E_{n}
\end{array}\right) .
$$

In the rest of the paper, we will refer to this model as to the most important example.

\subsection{Controllability and representation of the system with a graph}

To each system of the form (1), (H1) one can associate a topological graph (i.e. a set of points and a set of edges connecting the points) in a very natural way. The points are associated to the energy levels $E_{j}$ $(j=1, \ldots, n)$ and two points $E_{j}, E_{k}$ are connected by an edge iff the element $V_{j, k}$ is a control. This makes sense also if all the energy levels are zero (it happens after elimination of the drift, see Sect. 2).

Lifting the problem for the operator of temporal evolution $(i . e$. on $U(n))$ and with standard arguments of controllability on compact Lie groups and corresponding homogeneous spaces, one gets the following (see the recent survey [34] or the papers $[4,10,11,13,18,22,23])$ :

Proposition 1. The control system (1), under the assumption (H1) is completely controllable from any initial to any final condition if and only if the corresponding graph is connected.

In the following we deal with optimal control problems and we assume existence of minimizers for every couple of points. Hence we assume:

(H2) the graph associated with the control system (1), (H1) is connected.

If (H2) does not hold, then all the results of the paper are true for the restricted systems associated to the connected parts of the graph.

Remark 4. Notice that to guarantee controllability it is necessary (but not sufficient) to have at least $n-1$ controls $V_{j, k}$ (with $j<k$ ).

\subsection{The optimal control problem}

In this paper, we are faced with the problem of finding optimal trajectories for a convex cost depending only on the moduli of controls between a source and a target defined by conditions on the moduli of the components of the wave function. These are the most common situations in physics since the squares of moduli of the components of the wave function represent the probabilities of measures. More precisely our problem is the following:

Problem (P). Consider the control system (1), (H1), (H2) and assume that for time $t=0$ the state of the system is described by a wave function $\psi(0)$ whose components satisfy $\left(\left|\psi_{1}(0)\right|^{2}, \ldots,\left|\psi_{n}(0)\right|^{2}\right) \in S_{i n}$, where $S_{\text {in }}$ is a subset of the set:

$$
\mathbb{S}:=\left\{\left(a_{1}, \ldots, a_{n}\right) \in\left(\mathbb{R}^{+}\right)^{n}: \sum a_{i}=1\right\} .
$$

We want to determine suitable controls $V_{j, k}(), j,. k=1, \ldots, n$, defined on an interval $[0, T]$, such that for time $t=T$, the system is described by the wave function $\psi(T)$ satisfying $\left(\left|\psi_{1}(T)\right|^{2}, \ldots,\left|\psi_{n}(T)\right|^{2}\right) \in S_{\text {fin }}$, where 
$S_{\text {fin }} \subset \mathbb{S}$, requiring that these controls minimize a cost that depends only on the moduli of controls:

$$
\int_{0}^{T} f^{0}(V(t)) \mathrm{d} t
$$

Remark 5. Typical sources and targets are eigenstates. For instance if the source and the target are respectively the eigenstates 1 and $n$, we have $S_{i n}=(1,0, \ldots, 0), S_{\text {fin }}=(0,0, \ldots, 1)$.

In the following to guarantee the existence of minimizers we assume:

(H3) the function $f^{0}$ (that depends only on the moduli of controls) is convex. $S_{i n}$ and $S_{f i n}$ are closed subsets of $\mathfrak{S}$. Moreover the moduli of controls are bounded: there exist constants $M_{j, k} \geq 0$ such that $\left|V_{j, k}(t)\right| \leq M_{j, k}$, for every $t \in[0, T]$.

Remark 6. In the problem $(\mathbf{P})$, the final time can be fixed or free depending on the problem and on the explicit form of $f^{0}$ (see below).

Remark 7. Notice that if $f^{0}$ is convex and depends only on the moduli of controls then it is an increasing function (as function of the moduli of controls). Hypotheses (H3) could be relaxed, in particular if we do not require existence of minimizers for each couple of points, it is not always necessary to assume that controls are bounded or that $f^{0}$ is convex. Moreover the hypotheses of boundedness of controls could be changed with suitable hypotheses of growing of $f^{0}$ at infinity. Anyway these investigations are not the purpose of the paper. The costs on which we are interested are those described in the next Section, that are convex (some of them strictly). Moreover the corresponding minimization problems are always equivalent to minimization problems with bounded controls.

In the following if $V($.$) is a function satisfying (H1), (H2), (H3), and \psi($.$) the corresponding absolutely$ continuous trajectory we say that the couple $(\psi(),. V()$.$) is an admissible pair.$

\subsection{The interesting costs}

In this paper, we consider only costs that depend on the moduli of controls, since these are the interesting costs from a physical point of view, like those described in the following.

\section{Energy}

$$
\int_{0}^{T} f^{0} \mathrm{~d} t, \quad f^{0}=\sum_{j \leq k} \frac{1}{\mu_{j, k}^{2}}\left|V_{j, k}\right|^{2}
$$

This cost is proportional to the energy of the laser pulses. After elimination of the drift (see Sect. 2), the problem $(\mathbf{P})$, with this cost is a sub-Riemannian problem or a singular-Riemannian problem depending on the number of controls (see $(\mathrm{H} 1))$ and was studied in [10,11], in the isotropic case $\left(\mu_{j, k}=\mu\right)$, for $n=2,3$ and in the case in which the Hamiltonian is given by (2). For sub-Riemannian and singular-Riemannian geometry see for instance $[5,19,27]$. For this cost the final time must be fixed otherwise there are not solutions to the minimization problem. We recall that a minimizer for this cost is parameterized with constant velocity $\left(\sum_{j \leq k} \frac{1}{\mu_{j, k}^{2}}\left|V_{j, k}\right|^{2}=\right.$ const $)$ and it is also a minimizer for the sub-Riemannian length:

$$
\int_{0}^{T} f^{0} \mathrm{~d} t, \quad f^{0}=\sqrt{\sum_{j \leq k} \frac{1}{\mu_{j, k}^{2}}\left|V_{j, k}\right|^{2}}
$$

This cost (6) is invariant by re-parameterization, hence the final time $T$ can be equivalently fixed or free. For the costs $(5),(6)$ the controls are assumed to be unbounded. Anyway if the final time $T$ is fixed in such a way 
the minimizer is parameterized by arc-length $\left(\sum_{j \leq k} \frac{1}{\mu_{j, k}^{2}}\left|V_{j, k}\right|^{2}=1\right)$, then minimizing the cost (5) is equivalent to minimize time with moduli of controls constrained in the closed set:

$$
\sum_{j \leq k} \frac{1}{\mu_{j, k}^{2}}\left|V_{j, k}\right|^{2} \leq 1
$$

In the isotropic case $\mu_{j, k}=\mu=1$ the energy and the length are called respectively isotropic-energy and isotropic-length. They read:

$$
\begin{array}{r}
\int_{0}^{T} f^{0} \mathrm{~d} t, \quad f^{0}=\sum_{j \leq k}\left|V_{j, k}\right|^{2}, \\
\int_{0}^{T} f^{0} \mathrm{~d} t, \quad f^{0}=\sqrt{\sum_{j \leq k}\left|V_{j, k}\right|^{2}},
\end{array}
$$

and the set (7) becomes a closed ball. In the non-isotropic case we call (5) and (6) respectively non-isotropic-energy and non-isotropic-length.

\section{Isotropic and non-isotropic area}

$$
\left.\int_{0}^{T} f^{0} \mathrm{~d} t, \quad f^{0}=\sum_{j \leq k} \frac{1}{\mu_{j, k}}\left|V_{j, k}\right|, \quad \text { (isotropic case } \mu_{i}=\mu=1\right) .
$$

These costs are proportional to the area of the laser pulses $\int_{0}^{T} \sum_{j \leq k}\left|\mathcal{F}_{j, k}(t)\right| \mathrm{d} t$. They are invariant by reparameterization. Minimizing these costs is equivalent to minimize time with the following constraint on controls:

\section{Time with bounded controls}

$$
\sum_{j \leq k} \frac{1}{\mu_{j, k}^{2}}\left|V_{j, k}\right| \leq 1
$$

If we want to minimize time having a bound on the maximal amplitudes of the lasers, $\left|\mathcal{F}_{j, k}\right| \leq \nu_{j, k}(j \leq k)$ then we get the bounds on $V_{j, k}:\left|V_{j, k}\right| \leq \mu_{j, k} \nu_{j, k} / 2$. With the change of notation $\mu_{j, k} \nu_{j, k} / 2 \rightarrow \mu_{j, k}$ we get:

$$
\left|V_{j, k}\right| \leq \mu_{j, k}
$$

In this case we have $f^{0}=1$. Now the isotropic case $\mu_{j, k}=\mu=1$ corresponds to an isotropic system in which all the lasers have the same bound on the amplitude.

In this case the problem is equivalent (up to a re-parameterization) to minimize:

$$
\int_{0}^{T} f^{0} \mathrm{~d} t, \quad f^{0}=\max _{j \leq k}\left|\frac{V_{j, k}}{\mu_{j, k}}\right| .
$$

In the following figure the level sets $f^{0}=1$ for the $n=3$ case for the Hamiltonian (2) are drawn (of course in $\mathbb{R}^{2}$ instead of $\mathbb{C}^{2}$ ). If we consider the equivalent problems of minimizing time with constrained controls, then controls should lie in the compact regions whose borders are these level sets.

\subsection{Main questions}

Although the system (1), (H1) has a lot of symmetries, and good properties, the problem (P) in general is very difficult to solve also for the costs described above, for the reasons explained in the introduction: 
Isotropic Energy: $\mathrm{f}^{0}=\left|\mathrm{V}_{1,2}\right|^{2}+\left|\mathrm{V}_{2,3}\right|^{2} \quad$ Non-isotropic Energy: $\mathrm{f}^{0}=\frac{\left|\mathrm{V}_{1,2}\right|^{2}}{\mu_{1,2}^{2}}+\frac{\left|\mathrm{V}_{2,3}\right|^{2}}{\mu_{2,3}^{2}}$
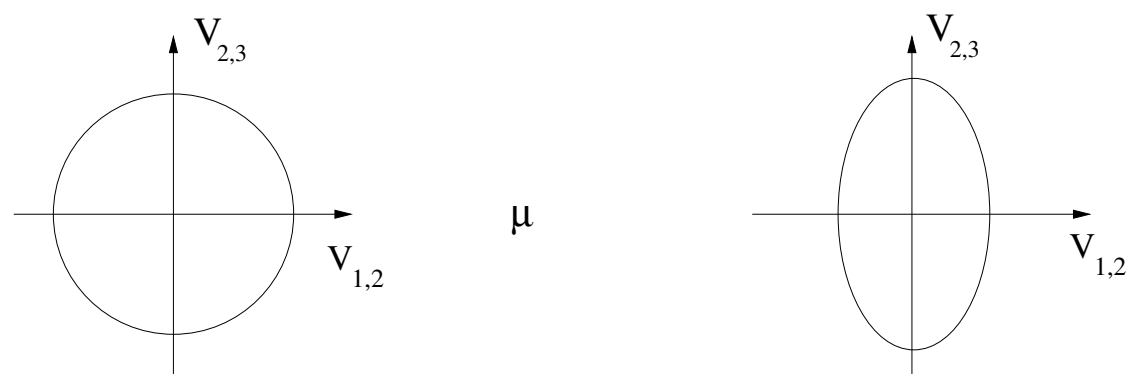

Isotropic Area: $\mathrm{f}^{0}=\left|\mathrm{V}_{1,2}\right|+\left|\mathrm{V}_{2,3}\right| \quad$ Non-isotropy Area: $\mathrm{f}^{0}=\frac{\left|\mathrm{V}_{1,2}\right|}{\mu_{1,2}}+\frac{\left|\mathrm{V}_{2,3}\right|}{\mu_{2,3}}$
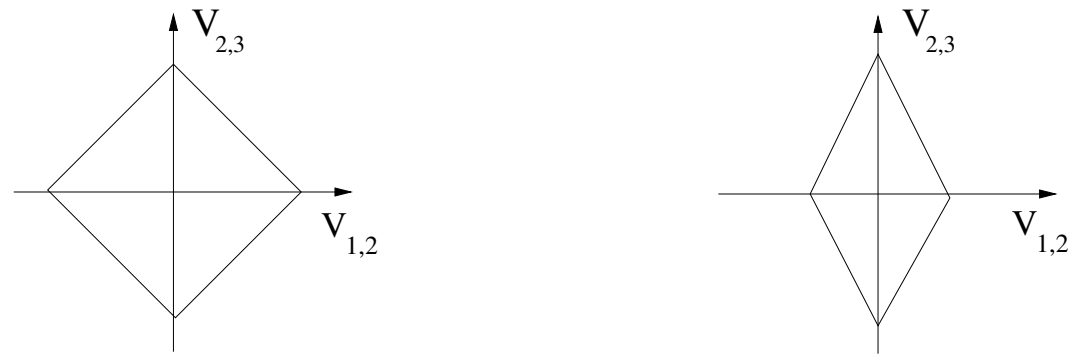

Time with b. contr: $f^{0}=\max \left(\left|V_{1,2}\right|,\left|V_{2,3}\right|\right) \quad$ Time with b. contr: $f^{0}=\max \left(\frac{\left|V_{1,2}\right|}{\mu_{1,2}}, \frac{\left|V_{2,3}\right|}{\mu_{2,3}}\right)$ (Isotropic Case)

(Non-isotropic Case)
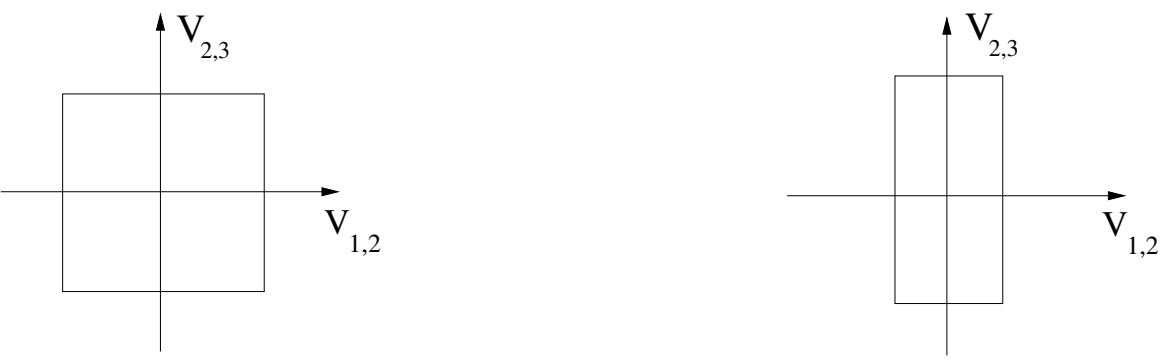

Figure 1. Level $f^{0}=1$ for different cost functions. 
- problem of integrability of the Hamiltonian system associated with the PMP;

- presence of abnormal extremals;

- problem of selecting the optimal trajectories among those satisfying the PMP.

Up to now the problem is solved for $n=2$ (for the costs described in Sect. 1.4, that, in this case, are all equivalent) and for $n=3$, for the Hamiltonian given by (2) for the isotropic energy (see [10,11]). In both cases optimal controls appear to be in resonance (with the difference between the energy levels that they are coupling) and every strictly abnormal extremal is not optimal. In this paper we generalize these results for more general systems and costs. Let us first introduce the definition of resonance and abnormal extremals:

Definition 1 (resonance). Consider the control system (1), (H1) and an admissible pair $(\psi(),. V()$.$) defined$ in an interval $[0, T]$. We say that the couple $(\psi(),. V()$.$) is in resonance (or is resonant) if the controls V_{j, k}($. have a.e. the form:

$$
\begin{aligned}
& V_{j, k}(t)=U_{j, k}(t) \mathrm{e}^{i\left[\left(E_{j}-E_{k}\right) t+\pi / 2+\varphi_{j, k}\right]}, \\
& \text { where: } U_{j, k}(.):[0, T] \rightarrow \mathbb{R}, U_{j, k}=-U_{k, j}, \\
& \varphi_{j, k}:=\arg \left(\psi_{j}(0)\right)-\arg \left(\psi_{k}(0)\right) \in[-\pi, \pi] .
\end{aligned}
$$

In formula (15), if $\psi_{j}(0)=0$, then arg $\left(\psi_{j}(0)\right)$ is intended to be an arbitrary number.

Remark 8. From a physical point of view, to be in resonance means to use lasers (described by complex functions) oscillating with a frequency given by $\omega /(2 \pi)$ where $\omega$ is the difference of energy between the levels that the laser is coupling. Notice that in formula (14) $U_{j, k}($.$) takes real values (it describes the amplitude of$ the lasers).

In the definition of resonance, the phases $\varphi_{j, k}$ sometimes are important and sometimes not, depending on the explicit form of the control term $V(t)$ in (1) and on the choice of the source and the target. For instance if we are considering the problem described by the Hamiltonian (2) and we start from an eigenstate $\left(e . g .\left|\psi_{1}(0)\right|=1\right)$ then all the phases $\varphi_{j, k}$ appearing in formula (13) are arbitrary. In fact in this case we have $n-1$ arbitrary numbers $\arg \left(\psi_{j}(0)\right)$ and $n-1$ controls. Therefore in this case it is not necessary to synchronize the phases of the lasers between them.

On the other side, if we start from a state that is not an eigenstate or we have more controls than the minimum number necessary to guarantee controllability (see Rem. 4), to be in resonance we need to synchronize the lasers according to formula (15).

In any case notice that a global factor of phase in front of $\psi$ is not important because it can be eliminated with a unitary transformation. Hence all the phases $\varphi_{j, k}$ can be shifted by an arbitrary factor $\alpha$.

Remark 9. To prove that a minimizer corresponds to controls in resonance, is very important because, as we will see after elimination of the drift, this permits us to reduce the dimension of the problem from the complex sphere $S^{2 n-1} \subset \mathbb{C}^{n}$ to the real sphere $S^{n-1} \subset \mathbb{R}^{n}$ (See the Cor. 1 in Sect. 3). This reduction of dimension is crucial in finding complete solutions to the optimal control problem in many cases. For instance for the class of problem described by the Hamiltonian (2):

A: an isotropic or non-isotropic minimum time problem with bounded controls for a 3-level system, is a problem in dimension 5. In this case, since the dimension of the state space is big, the problem of finding extremals and selecting optimal trajectories can be extremely hard. The possibility of proving that one can restrict to minimizers that are in resonance (and this is the case!) permits us to reduce the problem to a bi-dimensional problem, that can be solved with standard techniques (see for instance $[9,21,29,36])$;

B: a nonisotropic minimum energy problem for a 3-level system is naturally lifted to a left invariant subRiemannian problem on the group $S U(3)$. This problem cannot be solved with the techniques used in [11] for the isotropic case, because now the cost is built with a "deformed Killing form". Anyway if one can restrict to minimizers that are in resonance, the problem is reduced to a contact sub-Riemannian problem on $S O(3)$, that does not have abnormal extremals (since it is contact) and the corresponding 
Hamiltonian system is completely integrable (since it leads to a left invariant Hamiltonian system on a Lie group of dimension 3).

Items $\mathbf{A}$. and $\mathbf{B}$. are studied in a forthcoming paper.

C: An isotropic or nonisotropic minimum energy problem for a 4-level system is a problem on $S^{7}$ or can be lifted to a left invariant sub-Riemannian problem on $S U(4)$. In this case we conjecture that the Hamiltonian system given by the PMP is not Liouville integrable both before and after the reduction to real variables. Anyway this reduction can be crucial in looking for numerical solution to the PMP (it is of course easier making numerical computation on $S^{3}$ than on $S^{7}$ !). See [12] for some numerical solutions to this problem.

Moreover proving that there always exists a minimizer in resonance permits us to justify some strategies used in experimental physics.

To introduce the concept of abnormal extremals, we have to state the PMP, that is a necessary condition for optimality. The proof can be found for instance in $[1,21,31]$.

Theorem (Pontryagin Maximum Principle). Consider a control system of the form $\dot{x}=f(x, u)$ with a cost of the form $\int_{0}^{T} f^{0}(x, u) \mathrm{d} t$, and initial and final conditions given by $x(0) \in M_{i n}, x(T) \in M_{\text {fin }}$, where $x$ belongs to a manifold $M$ and $u \in U \subset \mathbb{R}^{m}$. Assume moreover that $M, f, f^{0}$ are smooth and that $M_{\text {in }}$ and $M_{\text {fin }}$ are smooth submanifolds of $M$. If the couple $(u(),. x()):.[0, T] \subset \mathbb{R} \rightarrow U \times M$ is optimal, then there exists a never vanishing field of covectors along $x($.$) , that is an absolutely continuous function \left(P(),. p_{0}\right): t \in[0, T] \mapsto$ $\left(P(t), p_{0}\right) \in T_{x(t)}^{*} M \times \mathbb{R}$ (where $p_{0} \leq 0$ is a constant) such that:

i) $\dot{x}(t)=\frac{\partial \mathscr{H}(}{\partial P}(x(t), P(t), u(t))$;

ii) $\dot{P}(t)=-\frac{\partial \mathscr{H}}{\partial x}(x(t), P(t), u(t))$,

where by definition:

$$
\mathscr{H}(x, P, u):=\langle P, f(x, u)\rangle+p_{0} f^{0}(x, u)
$$

Moreover:

iii) $\mathscr{H}(x(t), P(t), u(t))=\mathscr{H}_{M}(x(t), P(t))$, for a.e. $t \in[0, T]$; where $\mathcal{H}_{M}(x, P):=\max _{v \in U} \mathcal{H}(x, P, v)$;

iv) $\mathcal{H}_{M}(x(t), P(t))=k \geq 0$, where $k$ depends on the final time (if it is fixed) or $k=0$ if it is free;

v) $\left\langle P(0), T_{x(0)} M_{i n}\right\rangle=\left\langle P(T), T_{x(T)} M_{\text {fin }}\right\rangle=0$ (transversality conditions).

The real-valued map on $T^{*} M \times U$, defined in (16) is called PMP-Hamiltonian. A trajectory $x($.$) (resp. a$ couple $(u(),. x())$.$) satisfying conditions i), ii), iii) and iv) is called an extremal (resp. extremal pair.) If$ $x($.$) satisfies i), ii), iii) and iiii) with p_{0}=0$ (resp. $p_{0}<0$ ), then it is called an abnormal extremal (resp. a normal extremal). It may happen that an extremal $x($.$) is both normal and abnormal. So it makes sense to$ speak of strictly abnormal extremals (extremals that are abnormal but not normal).

Remark 10. Notice that the definition of abnormal extremal does not depend on the cost but only on the dynamics (in fact if $p_{0}=0$, the cost disappears in (16)). After elimination of the drift (see Sect. 2) our control system (1) will be transformed into a distributional system, that is a system of the form $\dot{x}=\sum u_{j} F_{j}(x)$. For these systems abnormal extremals are also singularities of the end point mapping, and have very special features (see for instance the recent monograph $[8]$ ).

Remark 11. In this paper we are dealing only with non-state dependent costs, i.e. $f^{0}(x, u)=f^{0}(u)$.

Consider the problem (P) under the assumption (H3). Hence a minimizer exists. The main purpose of this paper is to answer to the following questions: 
Q1: does there exist a minimizer that corresponds to controls in resonance?

Q2: once we have given a positive answer to question Q1, we would like to answer the question: are all the minimizers of the problem $(\mathbf{P})$ in resonance?

Q3: do there exist strictly abnormal minimizers?

The answer to question $\mathbf{Q 1}$ is yes, while $\mathbf{Q} 3$ is still an open question, although we are able to prove a result that strongly restricts the set of candidates strictly abnormal minimizers.

These results are formalized in theorems in Section 3.

The answer to question Q2 is in general no, but for an increasing cost we show that every minimizer is in weak-resonance (again this will be formalized in a theorem after elimination of the drift). To define this notion, in the next section we introduce some notations that will be also useful in Section 4 to answer Q1 and Q2.

Finally another interesting question for physicists is:

Q1': is it possible to join two arbitrary states $\psi^{1}$ and $\psi^{2}$ by an optimal trajectory in resonance?

The answer is, of course, in general, no. But there are couples of points for which the answer is yes. In particular if we consider eigenstates, it is true (see Sect. 4.2).

\subsubsection{Weak-Resonance}

To introduce this concept, we need some notations.

Consider an admissible pair $(\psi(),. V()$.$) and define the following subset of ] 0, T[$ :

$$
\operatorname{Bad}_{j, k}=\{t \in] 0, T\left[\text {, s.t. } \psi_{j}(t)=0 \text { or } \psi_{k}(t)=0\right\} .
$$

Since $\psi($.$) is continuous, the sets ] 0, T\left\lceil\backslash B a d_{j, k}\right.$ are open. Then they can be expressed as a countable union of open maximal intervals:

$$
] 0, T\left[\backslash \operatorname{Bad}_{j, k}=: \bigcup_{l} I_{j, k, l}, \quad j, k=1, \ldots, n, \quad l=1, \ldots m, \text { where } \underline{m \in\{0\} \cup \mathbb{N} \cup\{\infty\}} .\right.
$$

In other words $I_{j, k, l}$ are the maximal open intervals on which $\psi_{j}(t) \psi_{k}(t) \neq 0$.

Remark 12. In general $\overline{\cup_{l} I_{j, k, l}}$ is smaller than $[0, T]$ since it may happen that $\psi_{j}(t) \psi_{k}(t)=0$ on some interval of positive measure.

Now we are ready to give the following:

Definition 2 (weak-resonance). Consider the control system (1),(H1) and an admissible pair $(\psi(),. V()$.$) defined$ in an interval $[0, T]$. We say that the couple $(\psi(),. V()$.$) is in weak-resonance (or is weakly-resonant) if in each$ interval $I_{j, k, l}$ (defined above) the controls $V_{j, k}($.$) satisfy a.e.:$

$$
\begin{aligned}
& \left.V_{j, k}\right|_{I_{j, k, l}}(t)=U_{j, k, l}(t) e^{i\left[\left(E_{j}-E_{k}\right) t+\pi / 2+\varphi_{j, k, l}\right]}, \\
& \text { where: } U_{j, k, l}(.):[0, T] \rightarrow \mathbb{R}, \quad U_{j, k, l}=-U_{k, j, l} \\
& \varphi_{j, k, l}:=\arg \left(\psi_{j}\left(a_{j, k, l}\right)\right)-\arg \left(\psi_{k}\left(a_{j, k, l}\right)\right) \in[-\pi, \pi]
\end{aligned}
$$

where $\left.I_{j, k, l}=:\right] a_{j, k, l}, b_{j, k, l}\left[\right.$. In formula (21), if $\psi_{j}\left(a_{j, k, l}\right)=0$, then $\arg \left(\left(\psi_{j}\left(a_{j, k, l}\right)\right)\right)$ is intended to be an arbitrary number.

Remark 13. Roughly speaking a control $V_{j, k}($.$) is weakly-resonant if it is resonant in each interval of time in$ which the states that it is coupling (i.e. $\psi_{j}$ and $\psi_{k}$ ) are different from zero.

If the cost is not strictly increasing (for instance minimum time with bounded controls) in general there are minimizer that are not in weak-resonance, see Section 4.3 for an example and another open question. Finally notice that a resonant minimizer is also weakly-resonant. 


\section{Elimination OF THE DRIFT TERM}

The great advantage of the model presented in the previous section (in which each control is complex and couples only two levels) is that we can eliminate the drift term $D=\operatorname{diag}\left(E_{1}, \ldots, E_{n}\right)$ from equation (1) (hence getting a system in distributional form $\left.\dot{x}=\sum_{j} u_{j} F_{j}(x)\right)$, simply by using the interaction picture. This is made by a unitary change of coordinates and a unitary change of controls. Since the transformation is unitary, $S_{i n}$, $S_{\text {fin }}$ and the moduli of the components of the wave function are invariant. As a consequence the original and the transformed systems describe exactly the same population distribution.

Assume that $\psi(t)$ satisfies the Schrödinger equation (1). Let $\boldsymbol{U}(t)$ be a unitary time dependent matrix and set $\psi(t)=\boldsymbol{U}(t) \psi^{\prime}(t)$. Then $\psi^{\prime}(t)$ satisfies the Schrödinger equation:

$$
i \frac{\mathrm{d} \psi^{\prime}(t)}{\mathrm{d} t}=\mathcal{H}^{\prime}(t) \psi^{\prime}(t)
$$

with the new Hamiltonian:

$$
\mathcal{H}^{\prime}=\boldsymbol{U}^{-1} \mathcal{H} \boldsymbol{U}-i \mathcal{U}^{-1} \frac{\mathrm{d} \boldsymbol{U}}{\mathrm{d} t}
$$

If we choose:

$$
\mathcal{U}(t)=\mathrm{e}^{-i D t}
$$

and we recall that $\mathcal{H}=D+V(t)$, we get $\mathcal{H}^{\prime}=e^{i D t} V(t) \mathrm{e}^{-i D t}$, that is a Hamiltonian whose elements are either zero or can be redefined to be controls. Hence the drift is eliminated. Finally dropping the primes and including the $i$ in the new Hamiltonian $\left(\psi^{\prime} \rightarrow \psi, H:=-i \mathcal{H}^{\prime}\right)$ the Schrödinger equation reads:

$$
\frac{\mathrm{d} \psi(t)}{\mathrm{d} t}=H(t) \psi(t)
$$

Here $H$ is skew-Hermitian and its elements are either zero or controls. There is no more drift and the dynamics is in "distributional" form, i.e. $\dot{\psi}_{j}(t)=\sum_{k=1}^{n} H_{j, k}(t) \psi_{k}(t)$.

The relation between the old controls $V(t)_{j, k}$ (for Eq. (1)) and the new controls $H(t)_{j, k}$ for equation (24) is the following:

$$
V(t)_{j, k}=\left(i e^{-i D t} H(t) e^{i D t}\right)_{j, k}=H(t)_{j, k} e^{i\left[\left(E_{k}-E_{j}\right) t+\pi / 2\right]} .
$$

Remark 14. Notice that the transformation (23) kills also a not-only-diagonal drift. While to kill a time dependent drift we need the transformation (see [10]):

$$
\mathcal{U}(t)=\mathrm{e}^{-i \int_{t_{0}}^{t} D(t) \mathrm{d} t}
$$

\section{Statement of the MAIn RESUlts}

With the transformation described above (formula (23), and following), the problem (P) becomes the problem ( $\left.\mathbf{P}^{\prime}\right)$ below and the answers to questions Q1, Q2, Q3 are contained in the next Theorems, that we are going to prove in Sections 4, 5 . 
Problem ( $\left.\mathbf{P}^{\prime}\right)$. Consider the minimization problem:

$$
\begin{aligned}
& \frac{\mathrm{d} \psi(t)}{\mathrm{d} t}=H(t) \psi(t) \\
& \min \int_{0}^{T} f^{0}(H) \mathrm{d} t . \\
& \left(\left|\psi_{1}(0)\right|^{2}, \ldots,\left|\psi_{n}(0)\right|^{2}\right) \in S_{i n} \\
& \left(\left|\psi_{1}(T)\right|^{2}, \ldots,\left|\psi_{n}(T)\right|^{2}\right) \in S_{f i n}
\end{aligned}
$$

where:

- $f^{0}$ depends only on the moduli of controls;

- there hold (H1'), (H2), (H3). where (H1') is the following condition:

(H1') the matrix $H(t)$ is skew-Hermitian, measurable as function of $t$ and its elements are either identically zero or controls.

We have the following:

Theorem 1 (answer yes to Q1: existence of resonant minimizers). Let $(\psi(),. H()$.$) , defined in [0, T]$, be a solution to the minimization problem ( $\left.\mathbf{P}^{\prime}\right)$. Then there exists another solution $(\bar{\psi}(),. \bar{H}()$.$) , to the same minimization$ problem (with the same source, target and initial condition $\psi(0)=\bar{\psi}(0)$ ) that is in resonance:

$$
\begin{aligned}
& \bar{H}_{j, k}(t)=U_{j, k}(t) \mathrm{e}^{i \varphi_{j, k}}, \\
& \text { where: } U_{j, k}(.):[0, T] \rightarrow \mathbb{R}, \quad U_{j, k}=-U_{k, j} \\
& \varphi_{j, k}:=\arg \left(\psi_{j}(0)\right)-\arg \left(\psi_{k}(0)\right) \in[-\pi, \pi] .
\end{aligned}
$$

Moreover the arguments of $\bar{\psi}_{j}($.$) do not depend on the time. In formula (32), if \psi_{j}(0)=0$, then arg $\left(\psi_{j}(0)\right)$ is intended to be an arbitrary number.

Theorem 2 (answer to Q2). Let $(\psi(),. H()$.$) , defined in [0, T]$, be a solution to the minimization problem $\left(\mathbf{P}^{\prime}\right)$. Then if the cost is strictly increasing, $(\psi(),. H()$.$) is weakly-resonant:$

$$
\begin{aligned}
& \left.H_{j, k}(t)\right|_{I_{j, k, l}}=U_{j, k, l}(t) \mathrm{e}^{i \varphi_{j, k, l}}, \\
& \text { where }: U_{j, k, l}(.): I_{j, k, l} \rightarrow \mathbb{R}, \quad U_{j, k, l}=-U_{k, j, l} \\
& \varphi_{j, k, l}:=\arg \left(\psi_{j}\left(a_{j, k, l}\right)\right)-\arg \left(\psi_{k}\left(a_{j, k, l}\right)\right) \in[-\pi, \pi]
\end{aligned}
$$

where $\left.I_{j, k, l}=\right] a_{j, k, l}, b_{j, k, l}\left[\right.$ are the intervals defined in Section 1.5.1. In formula (35), if $\psi_{j}\left(a_{j, k, l}\right)=0$, then $\arg \left(\left(\psi_{j}\left(a_{j, k, l}\right)\right)\right)$ is intended to be an arbitrary number.

In the rest of the paper we call an admissible pair, a couple trajectory-control for an optimal control problem satisfying $\left(\mathrm{H} 1^{\prime}\right),(\mathrm{H} 2),(\mathrm{H} 3)$.

Remark 15. Notice that after elimination of the drift, to be in resonance implies to use controls with constant phases.

An important consequence of Theorem 1 is the following. If an admissible pair is a solution in resonance to the minimization problem, then there exists also one for which $\arg \left(\psi_{j}(0)\right)=0, j=1, \ldots, n$, i.e. corresponding to real controls and real initial conditions. In this case equation (26) restricts to reals, so $\psi(t) \in S^{n-1} \subset \mathbb{R}^{n}$. Hence it makes sense to give the following:

Definition 3. We call (RP'), the problem $\left(\mathbf{P}^{\prime}\right)$ in which all the coordinates and controls are real. 
And we have:

Corollary 1. If $(\psi(),. H()$.$) is a solution to the minimization problem (RP') (that is with \psi(t) \in S^{n-1} \in$ $\left.\mathbb{R}^{n}, H(t) \in \operatorname{so}(n)\right)$ then it is also a solution to the original problem $\left(\mathbf{P}^{\prime}\right)$ or equivalently, with the transformation described in Section 2, of the original problem $(\mathbf{P})$.

The following Theorem restricts the set of candidates strictly abnormal minimizers, for (RP'):

Theorem 3 (partial answer to $\mathrm{Q} 3$ for $\left.\left(\mathrm{RP}^{\prime}\right)\right)$. Let $(\psi(),. H()$.$) be a couple trajectory-control that is a minimizer$ for (RP') and assume that there are no constraints on the controls $\left(H(.)_{j, k}:\right.$ Dom $\left.(\psi) \rightarrow \mathbb{R}\right)$. Then for every $t \in \operatorname{Dom}(\psi)$, there exists an interval $\left[t_{1}, t_{2}\right]$ arbitrarily close to $t$ (possibly non containing $\left.t\right)$, on which $(\psi(),. H()$.$) is not a stricly abnormal extremal.$

For properties of abnormal extremals, we refer the reader to $[1,2,8,9,27,28]$.

Remark 16. In Theorem 3 the fact that we assume unconstrained controls is just a technical hypothesis that permits to simplify statements and proofs. Notice that for the costs described in Section 1.4 controls can always be assumed without constraints.

As we said above, the non existence of stricly abnormal minimizer is still a conjecture. After the result given by Theorem 3 to prove this conjecture one is essentially left with the following:

Problem. Let $(\psi(),. H()$.$) and (\bar{\psi}(),. \bar{H}()$.$) be two non strictly abnormal minimizers defined respectively on$ $\left[t_{a}, t_{b}\right]$ and $\left[t_{b}, t_{c}\right]$ with $t_{a}<t_{b}<t_{c}$. Is it true that the concatenation of the two is not strictly abnormal?

\section{ResonanCE AND WEAK-RESONANCE}

In this section we prove Theorems 1 and 2 . The key point is to identify the components of the control $H_{j, k}($. that are responsible of the evolution of $\left|\psi_{j}\right|$ and of $\arg \left(\psi_{j}\right)$. The difficulty is that these components are well defined only for the times such that $\psi_{j}(t) \neq 0$ and $\psi_{k}(t) \neq 0\left(\psi_{j}\right.$ and $\psi_{k}$ are the states coupled by $\left.H_{j, k}().\right)$. So we have to split the problem into the intervals $I_{j, k, l}$ defined in Section 1.5.1.

Let $(\psi(),. H()$.$) be a solution to the minimization problem and restrict the problem to the interval I_{\alpha}$, $\alpha=(j, k, l) \in(1, \ldots n)^{2} \times(1, \ldots, m)$, where $\psi_{j}$ and $\psi_{k}$ never vanish. Define in $I_{\alpha}$ :

$$
\begin{aligned}
\theta_{j}^{\alpha}(t) & :=\arg \left(\psi_{j}^{\alpha}(t)\right), \\
\rho_{j}^{\alpha}(t) & :=\left|\psi_{j}^{\alpha}(t)\right|, \\
\beta_{j, k}^{\alpha}(t) & :=\theta_{j}^{\alpha}(t)-\theta_{k}^{\alpha}(t) .
\end{aligned}
$$

From the definition of $I_{\alpha}$ the phases are well defined. Define the functions $u_{j, k}^{\alpha}(t)$ and $v_{j, k}^{\alpha}(t)$ in such a way that:

$$
H_{j, k}^{\alpha}(t):=\left.H_{j, k}(t)\right|_{I_{\alpha}}=:\left(u_{j, k}^{\alpha}(t)+i v_{j, k}^{\alpha}(t)\right) \mathrm{e}^{i \beta_{j, k}^{\alpha}(t)} .
$$

We have:

$$
\left\{\begin{array}{l}
u_{j, k}^{\alpha}=-u_{k, j}^{\alpha}, \quad v_{j, k}^{\alpha}=v_{k, j}^{\alpha} \\
\left|H_{j, k}^{\alpha}\right|=\left|u_{j, k}^{\alpha}+i v_{j, k}^{\alpha}\right|
\end{array}\right.
$$

Now in $I_{\alpha}$ :

$$
\dot{\psi}_{j}(t)=\sum_{k} H_{j, k}^{\alpha}(t) \psi_{k}(t)=\sum_{k}\left(u_{j, k}^{\alpha}(t)+i v_{j, k}^{\alpha}(t)\right)\left|\psi_{k}(t)\right| \mathrm{e}^{i \theta_{j}^{\alpha}(t)}
$$


From that, with a simple computation one gets:

$$
\begin{aligned}
\frac{\mathrm{d}}{\mathrm{d} t}\left(\rho_{j}^{\alpha}(t)\right) & =\sum_{k} u_{j, k}^{\alpha}(t) \rho_{k}^{\alpha}(t), \\
\frac{\mathrm{d}}{\mathrm{d} t}\left(\theta_{j}^{\alpha}(t)\right) & =\sum_{k} v_{j, k}^{\alpha}(t) \rho_{k}^{\alpha}(t) / \rho_{j}^{\alpha}(t) .
\end{aligned}
$$

These equations show that, on $I_{\alpha}, u_{j, k}^{\alpha}$ and $v_{j, k}^{\alpha}$ are responsible respectively of the evolution of $\left|\psi_{j}\right|$ and $\arg \left(\psi_{j}\right)$.

From the original minimizer $(\psi(),. H()$.$) we now define a new minimizer by means of two consecutive trans-$ formations:

- the first in which we set at zero all the $v^{\alpha}$-components of the control and we get a new admissible pair connecting $S_{i n}$ and $S_{\text {fin }}$ having a strictly smaller cost for a strictly increasing $f^{0}$ and that is weakly-resonant. This proves Theorem 2;

- the second in which $H_{j, k}$ is set to zero where $\psi_{j} \psi_{k}=0$ and in which all the phases $\beta_{j, k}^{\alpha}$ are set to $\arg \left(\psi_{j}(0)\right)-\arg \left(\psi_{k}(0)\right)$. This transformation does not increase the cost and produce a control in resonance. This proves Theorem 1 .

These transformations are realized in the following.

Let $\left(\psi(),. H(\right.$.$) be the original minimizer and let u_{j, k}^{\alpha}$ and $v_{j, k}^{\alpha}$ the corresponding components of the control in $I_{\alpha}$, defined in formula (36). Define a new admissible pair $(\bar{\psi}(),. \bar{H}()$.$) by setting in I_{\alpha}$ :

$$
\left\{\begin{array}{l}
\bar{u}_{j, k}^{\alpha}=u_{j, k}^{\alpha} \\
\bar{v}_{j, k}^{\alpha} \equiv 0 .
\end{array}\right.
$$

Notice the important point that $\bar{v}_{j, k}^{\alpha} \equiv 0$ implies that the corresponding $\bar{\beta}_{j, k}^{\alpha}$ are constants (see Eq. (39)) and they will be considered arbitrary phases. In other words $(\bar{\psi}(),. \bar{H}()$.$) is the admissible pair corresponding to the$ control:

$$
\bar{H}_{j, k}(t)=\left\{\begin{array}{l}
H_{j, k}(t) \text { if } t \notin \cup_{\alpha} I_{\alpha} \\
u_{j, k}^{\alpha}(t) \mathrm{e}^{i \bar{\beta}_{j, k}^{\alpha}} \text { if } t \in I_{\alpha} \text { for some } \alpha,
\end{array}\right.
$$

where $\bar{\beta}_{j, k}^{\alpha}$ are constant arbitrary phases. The corresponding trajectory $\bar{\psi}($.$) in each I_{\alpha}$ satisfies:

$$
\left\{\begin{array}{l}
\bar{\psi}_{j}(t)=\bar{\rho}_{j}^{\alpha}(t) \mathrm{e}^{i \bar{\theta}_{j}^{\alpha}} \\
\bar{\psi}_{k}(t)=\bar{\rho}_{k}^{\alpha}(t) \mathrm{e}^{i \bar{\theta}_{k}^{\alpha}}
\end{array}\right.
$$

with $\bar{\theta}_{j}^{\alpha}$ and $\bar{\theta}_{k}^{\alpha}$ constant and such that $\bar{\theta}_{j}^{\alpha}-\bar{\theta}_{k}^{\alpha}=\bar{\beta}_{j, k}^{\alpha}$. Now since $\bar{H}_{j, k}=H_{j, k}$ if $t \notin \cup_{\alpha} I_{\alpha}$ and the $u_{j, k}$ component is always the same for every $\alpha$, it follows that $\left|\bar{\psi}_{j}(t)\right|=\left|\psi_{j}(t)\right|$ for every $t \in[0, T]$. This means that $\psi($.$) and$ $\bar{\psi}($.$) connect the same source and target (recall that they are defined by conditions on moduli). By construction$ $(\bar{\psi}(),. \bar{H}()$.$) is in weak-resonance.$

Proof of Theorem 2.

By contradiction assume that:

- $f^{0}$ is strictly increasing;

- $(\psi(),. H()$.$) is not in weak-resonance that means v_{j, k}^{\alpha}(t) \neq 0$ on some set of positive measure $A \subset[0, T]$ and for some indexes $j, k, \alpha$.

In this case from equation (37) we have:

$$
\left|\bar{H}_{j, k}(t)\right|<\left|H_{j, k}(t)\right| \text { for each } \mathrm{t} \in A,
$$


that means:

$$
\int_{0}^{T} f^{0}(\bar{H}(t)) \mathrm{d} t<\int_{0}^{T} f^{0}(H(t)) \mathrm{d} t
$$

which contradicts the optimality of $(\psi(),. H()$.$) , proving Theorem 2$.

Proof of Theorem 1. In formula (41), the phases $\bar{\beta}_{j, k}^{\alpha}$ are arbitrary, so for each interval they could be chosen to be:

$$
\bar{\beta}_{j, k}^{\alpha}=\varphi_{j, k}:=\arg \left(\psi_{j}(0)\right)-\arg \left(\psi_{k}(0)\right) \in[-\pi, \pi]
$$

where $\arg \left(\psi_{j}(0)\right)$ is intended to be an arbitrary number if $\psi_{j}(0)=0$. This proves that if $(\psi(),. H()$.$) is a$ solution to the minimization problem, then there exists another solution $(\bar{\psi}(),. \bar{H}()$.$) that correspond to control$ $\bar{H}$ of the form $(30),(31),(32)$ on $\cup_{\alpha} I_{\alpha}$. It remains to consider the set $\left.B a d_{j, k}=\right] 0, T\left[\backslash \cup_{\alpha} I_{\alpha}\right.$, already defined in formula (17), where $\psi_{j}$ or $\psi_{k}$ are zero. The following lemma assures that on $B a d_{j, k}$, we can put the control at zero, without changing $\bar{\psi}$ and without increasing the cost.

Lemma 1. Let $(\psi(),. H()$.$) be an admissible pair and define a new control \hat{H}($.$) by:$

$$
\hat{H}_{j, k}(t):=\left\{\begin{array}{l}
H_{j, k}(t) \text { for all } t \text { such that } \psi_{j}(t) \psi_{k}(t) \neq 0 \\
0 \quad \text { otherwise. }
\end{array}\right.
$$

Then $(\psi(),. \hat{H}()$.$) is also an admissible pair and its cost is not bigger than the cost of (\psi(),. H()$.$) .$

Proof. Let us first prove that $\hat{H}($.$) is a measurable function. Let N_{j}$ be the set of times where $\psi_{j}$ is not 0 . Being $\psi_{j}($.$) measurable it follows that the function \mathbb{I}_{j}$ defined by $\mathbb{I}_{j}(t)=1$ if $t \in N_{j}, \mathbb{I}_{j}(t)=0$ if $t \notin N_{j}$, is measurable. This implies that $\hat{H}_{j, k}(t)=\mathbb{I}_{j}(t) \mathbb{I}_{k}(t) H_{j, k}(t)$ is measurable and $\hat{H}(t)$ as well. Let us now recall a classical:

Fact. Let $g():. \mathbb{R} \rightarrow \mathbb{R}$ be an absolutely continuous function. Then the set of points $x$ where $g(x)=0$ and $g^{\prime}(x) \neq 0$ has zero measure.

From this fact, we have a.e. that $\sum_{k} H_{j, k}(t) \psi_{k}(t)=\sum_{k} \hat{H}_{j, k}(t) \psi_{k}(t)$. Indeed, when $\psi_{k}(t)=0$ then $H_{j, k}(t) \psi_{k}(t)=\hat{H}_{j, k}(t) \psi_{k}(t)=0$ and for a.e. $t$ such that $\psi_{j}(t)=0$ we have $\dot{\psi}_{j}(t)=0$ and so $\sum_{k} H_{j, k}(t) \psi_{k}(t)=$ $0=\sum_{k} \hat{H}_{j, k}(t) \psi_{k}(t)$. Hence $H(t) \psi(t)=\hat{H}(t) \psi(t)$ for a.e. $t$ in [0,T]. It follows that if $\dot{\psi}(t)=H(t) \psi(t)$ a.e., then this relation holds also with $\hat{H}(t)$ in the place of $H(t)$, that is $(\psi(),. \hat{H}()$.$) is an admissible pair. From$ the fact that $\left|\hat{H}_{j, k}\right| \leq\left|H_{j, k}\right|$, it follows that $(\psi(),. \hat{H}()$.$) has a cost not bigger than (\psi(),. H()$.$) . The lemma is$ proved.

The proof of Theorem 1 is now complete.

Remark 17. In formula (43), $\bar{\beta}_{j, k}^{\alpha}$ has been chosen in such a way to be compatible with the initial condition:

$$
\bar{\psi}(0)=\psi(0)
$$

\subsection{Transversality}

In this section we give a more geometric interpretation to the proofs of Theorems 1 and 2 . We are going to show that the vector field associated to the control $v_{j, k}^{\alpha}$ defined in formula (36) is always tangent to a submanifold of $S^{2 n-1}$ whose points are reached with the same cost. As a consequence the transversality and maximum conditions of PMP say that the control $v_{j, k}^{\alpha}$ can (resp. must) be set to zero for a nondecreasing (resp strictly increasing) cost. 
Lemma 2. Given $\alpha_{1}, \ldots, \alpha_{n} \in[-\pi, \pi]$, let us define the map:

$$
\operatorname{Rot}_{\alpha}:\left\{\begin{aligned}
S^{2 n-1} & \rightarrow S^{2 n-1}, \\
\left(\psi_{1}, \ldots, \psi_{n}\right) & \mapsto\left(\mathrm{e}^{i \alpha_{1}} \psi_{1}, \ldots, \mathrm{e}^{i \alpha_{n}} \psi_{n}\right) .
\end{aligned}\right.
$$

If $\psi()=.\left(\psi_{1}(),. \ldots, \psi_{n}().\right)$ defined on $[0, T]$ is an admissible curve then $\operatorname{Rot}_{\alpha}(\psi()$.$) is also an admissible curve$ and has the same cost as $\psi($.$) .$

Proof. It is just a matter of computation. Let us denote by $H($.$) the control matrix associated to the admissible$ curve $\psi($.$) . The fact that \psi($.$) is an admissible curve, associated to the controls H($.$) , writes \dot{\psi}_{k}=\sum_{j} H_{j, k} \psi_{j}$, $\forall k \in\{1, \ldots, n\}$. By multiplying by $\mathrm{e}^{\mathrm{i} \alpha_{k}}$, we find $\dot{\psi}_{k} \mathrm{e}^{\mathrm{i} \alpha_{k}}=\sum_{j} H_{j, k} \mathrm{e}^{\mathrm{i}\left(\alpha_{k}-\alpha_{j}\right)} \psi_{j} \mathrm{e}^{\mathrm{i} \alpha_{j}}$. Hence the curve $R o t_{\alpha}(\psi)$ is an admissible curve corresponding to controls $\widetilde{H}_{j, k}=H_{j, k} \mathrm{e}^{\mathrm{i}\left(\alpha_{k}-\alpha_{j}\right)}$. And since the cost function does not depend on the phase of the controls, the two curves have the same cost.

This means that $\operatorname{Rot}_{\alpha}$ is an "isometry" for the cost defined by $f^{0}$, for any $\left(\alpha_{1}, \ldots, \alpha_{n}\right)$. A trivial consequence of this lemma is:

Corollary 2. For any two points $\psi^{1}$ and $\psi^{2}$ in $S^{2 n-1}$, all the points of the set:

$$
\mathcal{T}_{\psi^{2}}=\left\{\left(\psi_{1}^{2} \mathrm{e}^{i \alpha_{1}}, \ldots, \psi_{n}^{2} \mathrm{e}^{i \alpha_{n}}\right) \mid\left(\alpha_{1}, \ldots, \alpha_{n}\right) \in \mathbb{R}^{n}\right\}
$$

are reached with the same cost from the set:

$$
\mathcal{T}_{\psi^{1}}=\left\{\left(\psi_{1}^{1} \mathrm{e}^{i \alpha_{1}}, \ldots, \psi_{n}^{1} \mathrm{e}^{i \alpha_{n}}\right) \mid\left(\alpha_{1}, \ldots, \alpha_{n}\right) \in \mathbb{R}^{n}\right\}
$$

As a consequence if $\psi():.[0, T] \rightarrow S^{2 n-1}$ is a minimizing trajectory between the two sets $\mathcal{T}_{\psi^{1}} \mathcal{T}_{\psi^{2}}$, then the transversality condition of PMP (see v)) is:

$$
<P(t), T \mathcal{T}_{\psi(t)}>=0
$$

Now let $F_{j, k}^{\alpha}(\psi)$ and $G_{j, k}^{\alpha}(\psi)$ defined in $I_{j, k}^{\alpha}$ be the two vector fields associated with the controls $u_{j, k}^{\alpha}$ and $v_{j, k}^{\alpha}$ defined in formula $(36)$ :

One can easily check that on $I_{\alpha}$ :

$$
\dot{\psi}_{j}=\sum_{k}\left(u_{j, k}^{\alpha} F_{j, k}^{\alpha}(\psi)+v_{j, k}^{\alpha} G_{j, k}^{\alpha}(\psi)\right)
$$

$$
\begin{aligned}
& F_{j, k}^{\alpha}(\psi)=\mathrm{e}^{\mathrm{i} \beta_{j, k}^{\alpha}} \psi_{k} \frac{\partial}{\partial \psi_{j}}-\mathrm{e}^{-\mathrm{i} \beta_{j, k}^{\alpha}} \psi_{j} \frac{\partial}{\partial \psi_{k}}, \\
& G_{j, k}^{\alpha}(\psi)=i\left(\mathrm{e}^{\mathrm{i} \beta_{j, k}^{\alpha}} \psi_{k} \frac{\partial}{\partial \psi_{j}}+\mathrm{e}^{-\mathrm{i} \beta_{j, k}^{\alpha}} \psi_{j} \frac{\partial}{\partial \psi_{k}}\right) .
\end{aligned}
$$

And with a simple computation one can see that the vector $G_{j, k}^{\alpha}(\psi(t))$ is tangent to the set $\mathcal{T}_{\psi(t)}$ :

$$
G_{j, k}^{\alpha}(\psi(t)) \in T \mathcal{T}_{\psi(t)}, \quad \forall t \in I_{\alpha} .
$$

From (45) and (46), one get that $\left\langle P(t), G_{j, k}^{\alpha}(\psi(t))\right\rangle=0$. Then the maximality condition iii) of PMP implies (here $\left.f=u_{j, k}^{\alpha} F_{j, k}^{\alpha}(\psi)+v_{j, k}^{\alpha} G_{j, k}^{\alpha}(\psi)\right)$ :

$\sum_{j, k} u_{j, k}^{\alpha}(t)\left\langle P(t), F_{j, k}^{\alpha}(\psi(t))\right\rangle+p_{0} f^{0}\left(\left|u_{j, k}^{\alpha}(t)+i v_{j, k}^{\alpha}(t)\right|\right)=\max _{\bar{u}_{j, k}, \bar{v}_{j, k}} \sum_{j, k} \bar{u}_{j, k}\left\langle P(t), F_{j, k}^{\alpha}(\psi(t))\right\rangle+p_{0} f^{0}\left(\left|\bar{u}_{j, k}+i \bar{v}_{j, k}\right|\right)$

Now since $p_{0}<0$ (there are no strictly abnormal extremal, as it will be proved in Sect. 5 ), we get that (we stress the fact that $v_{j, k}^{\alpha}$ is not useful to reach the final target since the source and the target are defined by conditions on the moduli and $G_{j, k}^{\alpha}$ is responsible only of the evolution of the phases, see Eq. (39)): 
- for a non decreasing cost this condition is realized if $v_{j, k}^{\alpha}=0$ a.e. (plus conditions on $u_{j, k}^{\alpha}$ );

- for a strictly increasing cost this condition can be realized only if $v_{j, k}^{\alpha}=0$ a.e.

\subsection{Eigenstates}

Let us recall that an eigenstate is a state for which one of the coordinates has norm 1 and the others are 0 .

In this section we show that it is possible to join every couple of eigenstates by a minimizing trajectory that is in resonance (question Q1'). More in general we prove that the answer to question Q1' is yes for any couple of initial and final states $\psi^{1}=\left(\psi_{1}^{1}, \ldots, \psi_{n}^{1}\right)$ and $\psi^{2}=\left(\psi_{1}^{2}, \ldots, \psi_{n}^{2}\right)$ such that $\psi_{j}^{1} \psi_{j}^{2}=0$ for any $j$, that in particular is true for eigenstates.

Let $\bar{\psi}($.$) be a minimizing trajectory in resonance between the points \left(\left|\psi_{1}^{1}\right|, \ldots,\left|\psi_{n}^{1}\right|\right)$ and $\left(\left|\psi_{1}^{2}\right|, \ldots,\left|\psi_{n}^{2}\right|\right)$. Define $\theta_{j}^{1}$ and $\theta_{j}^{2}$ as the arguments of $\psi_{j}^{1}$ and $\psi_{j}^{2}$, putting them to 0 when the corresponding coordinate is 0 . We define $\alpha_{j}$ to be equal to $\theta_{j}^{1}$ if $\psi_{j}^{1} \neq 0$, or to $\theta_{j}^{2}$ if $\psi_{j}^{2} \neq 0$ and 0 if both $\psi_{j}^{1}$ and $\psi_{j}^{2}$ are 0 . Finally let $\alpha:=\left(\alpha_{1}, \ldots, \alpha_{n}\right)$. The curve

$$
\psi(.):=\operatorname{Rot}_{\alpha}(\bar{\psi}(.))
$$

is a resonant minimizer for the problem with initial and final condition $\psi^{1}$ and $\psi^{2}$.

\subsection{An example of a non-weakly-resonant minimizer}

In this section, in the case of a non strictly increasing cost, we show an example of an optimal couple trajectory-control $(\psi(),. H()$.$) , joining a source and a target defined by conditions on the moduli, that is$ neither resonant nor weakly-resonant. Consider a time minimization problem for a 4-level system in the form (2) (i.e. with controls on the lower and upper diagonal), for the isotropic case. We have $f^{0}(H)=$ $\max \left\{\left|H_{1,2}\right|,\left|H_{2,3}\right|,\left|H_{3,4}\right|\right\}$, that is not a strictly increasing function but just a not decreasing one.

It is easy to see that the following curve: $t \mapsto(\cos (t), \sin (t), 0,0)$ for $t \in\left[0, \frac{\pi}{2}\right]$ is a minimizer between the eigenstates $\left|\psi_{1}\right|=1$ and $\left|\psi_{2}\right|=1$. It can be obtained by different control functions. For instance by $t \mapsto(-1,0,0)$, or by $t \mapsto\left(-1,0, U_{3}\right)$ where

$$
\begin{array}{ll}
U_{3}(t)=1 & \text { for } t \in\left[0, \frac{\pi}{8}\right], \\
U_{3}(t)=-1 & \text { for } t \in\left[\frac{\pi}{8}, \frac{\pi}{4}\right], \\
U_{3}(t)=i & \text { for } t \in\left[\frac{\pi}{4}, \frac{3 \pi}{8}\right], \\
U_{3}(t)=-i & \text { for } t \in\left[\frac{3 \pi}{8}, \frac{\pi}{2}\right] .
\end{array}
$$

The second one is clearly not weakly-resonant.

The following is still an open question: under the hypothesis (H1'), (H2) and (H3), does there exist an example of non-decreasing cost function such that there exists a trajectory $(\psi(),. H()$.$) , solution of (P'), being$ non-weakly-resonant, such that there is no weakly-resonant control $\bar{H}$ with $(\psi(),. \bar{H}()$.$) solution of \left(\mathbf{P}^{\prime}\right)$ ?

\section{Strictly abNormal Minimizers For the REAL Problem}

In this section we prove Theorem 3 (i.e. we prove that close to any time $t$ of the domain of a given minimizer, there exist an interval $\left[t_{1}, t_{2}\right]$ where the minimizer is not strictly abnormal). We are able to prove the result only in such a interval, since on that interval we can make a suitable partition of indexes, see Lemma 3 (how to extend this result to the whole domain of the minimizer is still an open question, $c f$. end of Sect. 3). Then the difficulty of the proof is coming from the fact that the dynamics has singularities each time a coordinate is zero. For instance, in the most important example (see formula (2)), when $\psi_{1}=\psi_{2}=0$ then the control $V_{1,2}$ has no effect on the dynamics, i.e. the corresponding vector field is zero when $\psi_{1}=\psi_{2}=0$. Hence, in order to prove the theorem, we show (Sects. 5.1 and 5.2) that every minimizer $\widetilde{\psi}$ of the problem (RP') is also a solution of an auxiliary optimal problem (RP"), living on a submanifold $\mathcal{J}$ of $S^{n-1}$, which has no singularities. Then in Section 5.3 we prove that this new problem has no abnormal extremals (which proves that the minimizer $\widetilde{\psi}$ 
is a normal extremal as solution of (RP")). Finally, we come back to the original problem (RP') proving that the minimizer has a normal lift in $T^{*} S^{n-1}$ (Sect. 5.4).

In the following, let $(\widetilde{\psi}(),. \widetilde{H}()$.$) be a couple trajectory-control that is a minimizer for (RP').$

\subsection{Permutation of indexes}

In this section, we construct a submanifold $\mathcal{J}$ on which we will restrict the optimal problem (RP').

Lemma 3. In every neighborhood of every time $t$ of the domain of the minimizer, there exists a sub-interval of time, denoted by $\left[t_{1}, t_{2}\right]$ (possibly non containing $t$ ), such that there exists a partition $I \cup J$ of $\{1, \ldots, n\}$ satisfying:

- if $j \in I$ then $\psi_{j}(t)=0 \forall t \in\left[t_{1}, t_{2}\right]$;

- if $j \in J$ then $\psi_{j}(t) \neq 0 \forall t \in\left[t_{1}, t_{2}\right]$.

This is just a consequence of the continuity of $\psi($.$) . The proof is left to the reader. In the following we always$ restrict to the interval $\left[t_{1}, t_{2}\right]$.

Definition 4. We say that two indexes $j$ and $k$ of $J$ are connected (denoted by $j \sim k$ ) if $j=k$ or there exists a sequence $j_{1}, \ldots, j_{s}$ of indexes of $J$ such that $j=j_{1}, k=j_{s}$ and $\forall r<s$ the coefficient $H_{j_{r}, j_{r+1}}$ is a control.

Remark 18. This definition of connectedness is exactly the same as in Section 1.2 but for the sub-graph defined by $J$. Until the end of this section, "connected" refers to this last definition.

We immediately get:

Lemma 4. In $J$, the relation " $\sim$ " is an equivalence relation.

Definition 5. We denote $K_{1}, \ldots, K_{r}$ the equivalence classes defined by " $\sim$ " and $m_{1}, \ldots, m_{r}$ their cardinalities. We also denote $M_{0}=0$ and $M_{\ell}=\sum_{k \leq \ell} m_{k}$.

Now, let us make a permutation on the indexes that orders the sets $K_{1}, \ldots, K_{r}, I$ in such a way that:

$$
\forall \ell \leq r \quad K_{\ell}=\left\{M_{\ell-1}+1 ; \ldots ; M_{\ell}\right\}
$$

and

$$
I=\left\{M_{r}+1 ; \ldots ; n\right\}
$$

Lemma 5. After the permutation, we have:

a) for all $j \geq M_{r}+1(j \in I), \psi_{j} \equiv 0$ on $\left[t_{1}, t_{2}\right]$;

b) for all $\ell \leq r$, the map $t \mapsto \sum_{j \in K_{\ell}}\left|\psi_{j}(t)\right|^{2}=\sum_{j=M_{\ell-1}+1}^{M_{\ell}}\left|\psi_{j}(t)\right|^{2}$ is constant on $\left[t_{1}, t_{2}\right]$.

Proof. The point $\mathbf{a}$ ) is just a consequence of the definition of $I$. In order to prove the point $\mathbf{b}$ ), let us consider $\ell \leq r$. For any $j$ in $K_{\ell}$, we have:

$$
\dot{\widetilde{\psi}}_{j}(t)=\sum_{k \leq n} \widetilde{H}_{j, k}(t) \widetilde{\psi}_{k}(t)
$$

But $\widetilde{H}_{j, k} \equiv 0$ if $k \notin K_{\ell} \cup I$ and $\widetilde{\psi}_{k} \equiv 0$ if $k \in I$. Hence for $t$ in $\left[t_{1}, t_{2}\right]$ :

$$
\dot{\widetilde{\psi}}_{j}(t)=\sum_{k \in K_{\ell}} \widetilde{H}_{j, k}(t) \widetilde{\psi}_{k}(t)
$$

Now, the matrix $\widetilde{H}^{\ell}(t)$, whose coefficients are the $\widetilde{H}_{j, k}(t)$ with $j, k \in K_{\ell}$, belongs to $s o\left(m_{\ell}\right)$. Hence the vector $\widetilde{\psi}^{\ell}$, whose coefficients are the $\widetilde{\psi}_{j}(t)$ with $j \in K_{\ell}$, satisfies $\dot{\widetilde{\psi}}=\widetilde{H}^{\ell} \widetilde{\psi}^{\ell}$ and then has constant norm, i.e. $\sum_{j \in K_{\ell}}\left|\widetilde{\psi}_{j}(t)\right|^{2}$ is constant. 
From the previous Lemma 5 , it follows that for $t \in\left[t_{1}, t_{2}\right], \psi(t)$ belongs to the set:

$$
\mathcal{J}=S^{m_{1}-1}\left(C_{1}\right) \times \ldots \times S^{m_{r}-1}\left(C_{r}\right) \times \prod_{j \in I}\left\{\psi_{j}\left(t_{1}\right)\right\}
$$

where $C_{\ell}=\sqrt{\sum_{j \in K_{\ell}}\left|\psi_{j}\left(t_{1}\right)\right|^{2}}$.

Remark 19. In the following, we are going to restrict (RP') to $\mathcal{J}$. Notice that the dimension of $\mathcal{J}$ is $\sum_{\ell \leq r}\left(m_{\ell}-1\right) \leq n-1$, while the original problem $\left(\mathbf{R} \mathbf{P}^{\prime}\right)$ lives on $S^{n-1}$ that has dimension $n-1$.

\subsection{Restriction of the control system to $\mathcal{J}$}

In this Section, we show that $\widetilde{\psi}$ is also a solution of an auxiliary optimal problem (RP") in $\mathcal{J}$.

Definition 6. We call (RP") the optimal problem (RP') in which we restrict the dynamics by adding a new condition on the matrix of controls: $H_{j, k}(t)$ is set to 0 if $j$ or $k$ is in $I$.

Remark 20. Notice that $\mathcal{J}$ is preserved by the dynamics of (RP").

Lemma 6. The curve $\widetilde{\psi}($.$) is a minimizer for (RP")).$

Proof. As proved in Lemma $1, \widetilde{\psi}$ does not change if we set to zero the controls $\widetilde{H}_{j, k}$ such that one index belongs to $I$. Hence $\widetilde{\psi}$ is an admissible curve for (RP").

Now, since the cost function is the same for both $\left(\mathbf{R} \mathbf{P}^{\prime}\right)$ and (RP"), it follows that $\widetilde{\psi}$ is a minimizer for $(\mathbf{R P} ")$.

\subsection{The minimizer $\widetilde{\psi}($.$) is not an abnormal extremal for (RP")$}

Let us denote $F_{j, k}(\psi)$ the vector field associated with the control $H_{j, k}: \dot{\psi}=\sum_{j, k} H_{j, k} F_{j, k}(\psi)$. We denote by $\Delta_{\ell}$ the distribution generated by the $F_{j, k}(\psi)$ with $j, k \in K_{\ell}$ and $\Delta=\oplus_{\ell} \Delta_{\ell}$. We have that $\Delta(q) \subset T_{q} \mathcal{J}$ for $q \in \mathcal{J}$.

Using $F_{j, k}(\psi)$, the dynamics of (RP") reads:

$$
\dot{\psi}=\sum_{\ell} \sum_{j, k \in K_{\ell}} H_{j, k} F_{j, k}(\psi)
$$

Lemma 7. The distribution $\Delta$ is the whole tangent space of $\mathcal{J}: \Delta=T \mathcal{J}$.

Proof. Let us first prove that, for every $\ell, \Delta_{\ell}=T S^{m_{\ell}-1}\left(C_{\ell}\right)$.

First step: it exists a set $L_{\ell}$ of couples of indexes of $K_{\ell}$, with cardinality $m_{\ell}-1$, that keeps $K_{\ell}$ connected in the following sense: for any couple of indexes $j, k$ in $K_{\ell}$, there is a sequence $j_{1}, \ldots, j_{s}$ in $K_{\ell}$ such that $j_{1}=j$, $j_{s}=k,\left\{j_{p}, j_{p+1}\right\} \in L_{l}$ and $H_{j_{p}, j_{p+1}}$ is a control.

The proof can be done by induction on the cardinality of $K_{\ell}$. If it has cardinality one, $L_{\ell}$ is empty. If it has cardinality two, the proof is trivial. If the cardinality of $K_{\ell}$ is bigger than two, we can choose an index $k \in K_{\ell}$ such that $\widetilde{K}_{\ell}:=K_{\ell}-\{k\}$ is still connected (this is a standard fact of graph theory). Now, $\widetilde{K}_{\ell}$ is connected and has cardinality $m_{\ell}-1$. Hence, by induction hypothesis, there exists an $\widetilde{L}_{\ell}$ that keeps $\widetilde{K}_{\ell}$ connected and has cardinality $m_{\ell}-2$. Now, $k$ is connected to an index $j$ of $\widetilde{K}_{\ell}$, hence the set $L_{\ell}=\widetilde{L}_{\ell} \cup\{\{j, k\}\}$ keeps $K_{\ell}$ connected and has the required cardinality.

Second step: the family of $F_{j, k}(\psi)\left(\{j, k\} \in L_{\ell}\right)$ is linearly independent ( $L_{\ell}$ was constructed for this purpose).

The proof can be done by induction: if $m_{\ell}=1, L_{\ell}$ is empty. If $m_{\ell}>1$, then one can choose a index $k$ appearing only once in $L_{\ell}$. Let call $j$ the index such that $\{j, k\} \in L_{\ell}$. The vector $F_{j, k}(\psi)$ is linearly independent of the rest of the family: it is the only one vector that has a non zero $k$-coordinate. 
Now $L_{\ell}-\{\{j, k\}\}$ has cardinality $m_{\ell}-2$ and keeps connected $K_{\ell}-\{k\}$ of cardinality $m_{\ell}-1$. Hence we can apply induction.

Last step: from the previous step, it follows that $\Delta_{\ell}$ has the same cardinality $m_{\ell}-1$ as the sphere $S^{m_{\ell}-1}\left(C_{\ell}\right)$, hence we get $\Delta_{\ell}=T S^{m_{\ell}-1}\left(C_{\ell}\right)$.

Thus we have $\Delta=\oplus_{\ell} \Delta_{\ell}=\oplus_{\ell} T S^{m_{\ell}-1}\left(C_{\ell}\right)=T \mathcal{J}$.

Since the distribution $\Delta$ is the whole tangent space of $\mathcal{J}$, it is a standard fact that there are no abnormal extremals for the problem (RP").

\subsection{End of the proof}

Now, we are going to prove that the curve $\widetilde{\psi}($.$) admits a normal lift on S^{n-1}$ i.e. it is not a strictly abnormal extremal for the problem (RP').

Let us denote $\Theta_{\ell}$ a local coordinate system on $S^{m_{\ell}-1}\left(C_{\ell}\right)$, and $\Theta=\left(\Theta_{1}, \ldots, \Theta_{r}\right)$. Then:

$$
\left(\Theta_{1}, \ldots, \Theta_{r}, C_{1}, \ldots, C_{r}, \psi_{M_{r+1}}, \ldots, \psi_{n}\right)
$$

is a local coordinate system on $\mathbb{R}^{n}$. We denote by $P_{\Theta_{\ell}}, P_{C_{\ell}}$ and $P_{\psi_{j}}$ the dual coordinates in $T^{*} \mathbb{R}^{n}$,

$$
P=\sum_{\ell \leq r} P_{\Theta_{\ell}} d \Theta_{\ell}+\sum_{\ell \leq r} P_{C_{\ell}} d C_{\ell}+\sum_{i \in I} P_{\psi_{i}} d \psi_{i}
$$

and

$$
P_{\mathcal{J}}=\sum_{\ell \leq r} P_{\Theta_{\ell}} d \Theta_{\ell}
$$

its restriction to $\mathcal{J}$.

Remark 21. Notice that the vector fields $F_{j, k}$ with $j, k$ in $K_{\ell}$, depend only on the $\Theta_{\ell}$ coordinates and that they annihilate $\mathrm{d} C_{s}(s \leq r)$ and $\mathrm{d} \psi_{i}(i \in I)$. This fact will be essential to conclude the proof.

The PMP-Hamiltonian associated with (RP") is:

$$
\mathscr{H}_{\mathcal{J}}\left(\Theta, H, P_{\mathcal{J}}, p_{0}\right)=P_{\mathcal{J}}\left(\sum_{j, k \in J} H_{j, k} F_{j, k}(\Theta)\right)+p_{0} f^{0}(H),
$$

where $f^{0}$ is the cost function. Since $\widetilde{\psi}$ is a normal extremal for (RP"), there is a lift $\left(\widetilde{\psi}, \widetilde{H}, \widetilde{P}_{\mathcal{J}}, \widetilde{p}_{0}\right)$, with $\widetilde{p}_{0} \neq 0$, that satisfies:

and the maximality condition:

$$
\begin{aligned}
\dot{\widetilde{\Theta}}_{\ell} & =\frac{\partial \mathscr{H}_{\mathcal{J}}}{\partial P_{\Theta_{\ell}}}\left(\widetilde{\Theta}, \widetilde{H}, \widetilde{P}_{\mathcal{J}}, \widetilde{p}_{0}\right) \\
\dot{\tilde{P}}_{\Theta_{\ell}} & =-\frac{\partial \mathcal{H U}_{\mathcal{J}}}{\partial \Theta_{\ell}}\left(\widetilde{\Theta}, \widetilde{H}, \widetilde{P}_{\mathcal{J}}, \widetilde{p}_{0}\right)
\end{aligned}
$$

$$
\mathcal{H}_{\mathcal{J}}\left(\widetilde{\psi}(t), \widetilde{H}(t), \widetilde{P}_{\mathcal{J}}(t), \widetilde{p}_{0}\right)=\max _{H}\left\{\mathcal{H}_{\mathcal{J}}\left(\widetilde{\psi}(t), H, \widetilde{P}_{\mathcal{J}}(t), \widetilde{p}_{0}\right)\right\}
$$

where the maximum is taken over the set of controls.

Now, the PMP-Hamiltonian associated with (RP') is:

$$
\mathscr{H}\left(\psi, H, P, p_{0}\right)=P\left(\sum_{j, k \leq n} H_{j, k} F_{j, k}\right)+p_{0} f^{0}(H) .
$$

To conclude the proof of Theorem 3 we need the following: 
Claim. Let us denote: $\widetilde{P}=\widetilde{P}_{\mathcal{J}}+\sum_{\ell \leq r} 0 \mathrm{~d} C_{\ell}+\sum_{i \in I} 0 \mathrm{~d} \psi_{i}$. Then $\left(\widetilde{\psi}, \widetilde{H}, \widetilde{P}, \widetilde{p}_{0}\right)$ is a normal lift of $\widetilde{\psi}$ satisfying the PMP for (RP').

Proof. Let us first remark that $\widetilde{P}$ satisfies $\widetilde{P}_{C_{\ell}}=\widetilde{P}_{\psi_{i}}=0$ for every $\ell \leq r$ and $i \in I$.

In order to prove the claim, we have to prove that the lift satisfies the Hamiltonian equations:

$$
\begin{aligned}
\dot{\widetilde{\psi}} & =\frac{\partial \mathcal{H}}{\partial P}\left(\widetilde{\psi}, \widetilde{H}, \widetilde{P}, \widetilde{p}_{0}\right) \\
\dot{\widetilde{P}} & =-\frac{\partial \mathcal{H}}{\partial \psi}\left(\widetilde{\psi}, \widetilde{H}, \widetilde{P}, \widetilde{p}_{0}\right)
\end{aligned}
$$

and the maximality condition:

$$
\mathcal{H}\left(\widetilde{\psi}(t), \widetilde{H}(t), \widetilde{P}(t), \widetilde{p}_{0}\right)=\max _{H}\left\{\mathcal{H}\left(\widetilde{\psi}(t), H, \widetilde{P}(t), \widetilde{p}_{0}\right)\right\}
$$

The equation (47) is always trivially satisfied (it is the dynamics).

Let us prove that (48) holds. Firstly, we have that:

$$
\dot{\widetilde{P}}_{\Theta_{\ell}}=-\frac{\partial \mathcal{H}_{\mathcal{J}}}{\partial \Theta_{\ell}}\left(\widetilde{\Theta}, \widetilde{H}, \widetilde{P}_{\mathcal{J}}, \widetilde{p}_{0}\right)=-\frac{\partial \mathcal{H}}{\partial \Theta_{\ell}}\left(\widetilde{\psi}, \widetilde{H}, \widetilde{P}, \widetilde{p}_{0}\right)
$$

Secondly,

$$
\frac{\partial \mathcal{H}}{\partial C_{\ell}}\left(\widetilde{\psi}, \widetilde{H}, \widetilde{P}, \widetilde{p}_{0}\right)=\widetilde{P}\left(\sum_{j, k \leq n} \widetilde{H}_{j, k} \frac{\partial}{\partial C_{\ell}}\left(F_{j, k}\right)\right)
$$

but $\widetilde{H}_{j, k}=0$ if $i$ or $k$ is in $I$ and, because of Remark $21, \frac{\partial}{\partial C_{\ell}}\left(F_{j, k}\right)=0$ when $j$ and $k$ are in $J$. Hence $\dot{\widetilde{P}}_{C_{\ell}}=0=-\frac{\partial \mathcal{H}}{\partial C_{\ell}}\left(\widetilde{\psi}, \widetilde{H}, \widetilde{P}, \widetilde{p}_{0}\right)$. The proof is the same for $\widetilde{P}_{\psi_{i}}$ for $i \in I$. Hence (48) is satisfied.

Let us now prove (49). We have that, if $j$, or $k$, is in $I$ then $F_{j, k}(\widetilde{\psi})$ is in $\operatorname{span}\left\{\frac{\partial}{\partial \psi_{i}} ; i \in I\right\}$. Indeed, $F_{j, k}(\widetilde{\psi})=\widetilde{\psi}_{k} \frac{\partial}{\partial \psi_{j}}-\widetilde{\psi}_{j} \frac{\partial}{\partial \psi_{k}}$, and then if $j \in I$, then $\widetilde{\psi}_{j}=0$ and $F_{j, k}(\widetilde{\psi})=\widetilde{\psi}_{k} \frac{\partial}{\partial \psi_{j}}$. Hence, because $\widetilde{P}_{\psi}=0$, we have that $\widetilde{P}\left(F_{j, k}\right)=0$ if $j$ or $k$ is in $I$ and we have that for any control $H$ :

$$
\begin{aligned}
\mathcal{H A}\left(\widetilde{\psi}, H, \widetilde{P}, \widetilde{p}_{0}\right) & =\widetilde{P}\left(\sum_{j, k \leq n} H_{j, k} F_{j, k}(\widetilde{\psi})\right)+\widetilde{p}_{0} f^{0}(H) \\
& =\widetilde{P}\left(\sum_{j, k \in J}^{j \in J} H_{j, k} F_{j, k}(\widetilde{\psi})\right)+\widetilde{p}_{0} f^{0}(H) \\
& =\widetilde{P}_{\mathcal{J}}\left(\sum_{j, k \in J} H_{j, k} F_{j, k}(\widetilde{\psi})\right)+\widetilde{p}_{0} f^{0}(H) \\
& =\mathcal{H H}_{\mathcal{J}}\left(\widetilde{\psi}, H, \widetilde{P}_{\mathcal{J}}, \widetilde{p}_{0}\right) .
\end{aligned}
$$

Now the fact that $\left(\widetilde{\psi}, \widetilde{H}, \widetilde{P}_{\mathcal{J}}, \widetilde{p}_{0}\right)$ satisfies the maximality condition for (RP") $)$ allows to conclude that $\left(\widetilde{\psi}, \widetilde{H}, \widetilde{P}, \widetilde{p}_{0}\right)$ satisfies the maximality condition for (RP').

Acknowledgements. The authors are grateful to Andrei Agrachev and Jean-Paul Gauthier for many helpful discussions. The authors would like also to thank the anonymous referee for some crucial remarks. 


\section{REFERENCES}

[1] A.A. Agrachev and Yu.L. Sachkov, Control Theory from the Geometric Viewpoint. Springer-Verlag, EMS (2004) 1-410.

[2] A.A. Agrachev and A.V. Sarychev, Sub-Riemannian metrics: minimality of abnormal geodesics versus subanaliticity. ESAIM: COCV 2 (1997) 377-448.

[3] C. Altafini, Controllability of quantum mechanical systems by root space decomposition of su(N). J. Math. Phys. 43 (2002) 2051-2062.

[4] R. El Assoudi, J.P. Gauthier and I.A.K. Kupka, On subsemigroups of semisimple Lie groups. Ann. Inst. H. Poincaré Anal. Non Linéaire 13 (1996) 117-133.

[5] A. Bellaiche, The tangent space in sub-Riemannian geometry. Sub-Riemannian geometry. Progr. Math. 144 (1996) 1-78.

[6] K. Bergmann, H. Theuer and B.W. Shore, Coerent population transfer among quantum states of atomes and molecules. Rev. Mod. Phys. 70 (1998) 1003-1025.

[7] V.G. Boltyanskii, Sufficient Conditions for Optimality and the Justification of the Dynamics Programming Principle. SIAM J. Control Optim. 4 (1996) 326-361.

[8] B. Bonnard and M. Chyba, The Role of Singular Trajectories in Control Theory. Springer, SMAI, Vol. 40 (2003).

[9] U. Boscain and B Piccoli, Optimal Synthesis for Control Systems on 2-D Manifolds. Springer, SMAI, Vol. 43 (2004).

[10] U. Boscain, G. Charlot, J.-P. Gauthier, S. Guérin and H.-R. Jauslin, Optimal Control in laser-induced population transfer for two- and three-level quantum systems. J. Math. Phys. 43 (2002) 2107-2132.

[11] U. Boscain, T. Chambrion and J.-P. Gauthier, On the K+P problem for a three-level quantum system: Optimality implies resonance. J. Dyn. Control Syst. 8 (2002) 547-572.

[12] U. Boscain, T. Chambrion and J.-P. Gauthier, Optimal Control on a $n$-level Quantum System, in Proc. of the 2nd IFAC Workshop on Lagrangian and Hamiltonian Methods for Nonlinear Control, Astolfi, Gordillo and van der Schaft Eds., Elsevier (2003).

[13] W.M. Boothby and E.N. Wilson, Determination of the transitivity of bilinear systems. SIAM J. Control Optim. 17 (1979) $212-221$.

[14] P. Brunovsky, Existence of Regular Syntheses for General Problems. J. Differ. Equations 38 (1980) 317-343.

[15] P. Brunovsky, Every Normal Linear System Has a Regular Time-Optimal Synthesis. Math. Slovaca 28 (1978) 81-100.

[16] D. D'Alessandro and M. Dahleh, Optimal control of two-level quantum systems. IEEE Trans. Automat. Control 46 (2001) 866-876.

[17] U. Gaubatz, P. Rudecki, M. Becker, S. Schiemann, M. Kulz and K. Bergmann, Population switching between vibrational levels in molecular beams. Chem. Phys. Lett. 149 (1988) 463.

[18] J.P. Gauthier and G. Bornard, Controlabilite des sytemes bilineaires. SIAM J. Control Optim. 20 (1982) 377-384.

[19] M. Gromov, Carnot-Carathéodory spaces seen from within. Sub-Riemannian geometry. Progr. Math. 144 (1996) 79-323.

[20] R.G. Hulet and D. Kleppner, Rydberg Atoms in "Circular" states. Phys. Rev. Lett. 51 (1983) 1430-1433.

[21] V. Jurdjevic, Geometric Control Theory. Cambridge University Press (1997).

[22] V. Jurdjevic and I.K. Kupka, Control Systems on Semisimple Lie Groups and Their Homogeneous Spaces. Ann. Inst. Fourier 31 (1981) 151-179.

[23] V. Jurdjevic and H.J. Sussmann, Controllability of Non-Linear systems. J. Differ. Equation 12 95-116.

[24] N. Khaneja, R. Brockett and S.J. Glaser, Time optimal control in spin systems. Phys. Rev. A 63 (2001).

[25] N. Khaneja and S.J. Glaser, Cartan decomposition of SU(n) and Control of Spin Systems. J. Chem. Phys. 267 (2001) 11-23.

[26] C. Liedenbaum, S. Stolte and J. Reuss, Inversion produced and reversed by adiabatic passage. Phys. Rep. 178 (1989) 1-24.

[27] R. Montgomery, A Tour of Subriemannian Geometry. American Mathematical Society, Mathematical Surveys and Monographs (2002).

[28] R. Montgomery, A survey of singular curves in sub-Riemannian geometry. J. Dyn. Control Syst. 1 (1995) 49-90.

[29] B. Piccoli, Classifications of Generic Singularities for the Planar Time-Optimal Synthesis. SIAM J. Control Optim. 34 (1996) 1914-1946.

[30] B. Piccoli and H.J. Sussmann, Regular Synthesis and Sufficiency Conditions for Optimality. SIAM. J. Control Optim. 39 (2000) 359-410.

[31] L.S. Pontryagin, V. Boltianski, R. Gamkrelidze and E. Mitchtchenko, The Mathematical Theory of Optimal Processes. John Wiley and Sons, Inc (1961).

[32] M.A. Daleh, A.M. Peirce and H. Rabitz, Optimal control of quantum-mechanical systems: Existence, numerical approximation, and applications. Phys. Rev. A $\mathbf{3 7}$ (1988).

[33] V. Ramakrishna, K.L. Flores, H. Rabitz and R.Ober, Quantum control by decomposition of su(2). Phys. Rev. A 62 (2000).

[34] Y. Sachkov, Controllability of Invariant Systems on Lie Groups and Homogeneous Spaces. J. Math. Sci. 100 (2000) $2355-2427$.

[35] B.W. Shore, The theory of coherent atomic excitation. New York, NY, Wiley (1990).

[36] H.J. Sussmann, The Structure of Time-Optimal Trajectories for Single-Input Systems in the Plane: the $C^{\infty}$ Nonsingular Case. SIAM J. Control Optim. 25 (1987) 433-465. 